\title{
Relationship between oxidative stress and inflammation in peripheral and cerebral system of oxonate-induced hyperuricemic rats
}

\author{
Wu Jing ${ }^{1,2}$, Jia Zhong ${ }^{1,2}$, Liang Jian Ping ${ }^{1 *}$, Liu Hong Yan ${ }^{2}$ \\ ${ }^{1}$ College of Veterinary Medicine, Gansu Agricultural University, LanZhou, ${ }^{2}$ Department of Pharmacy, Pulmonary Hospital of \\ Lanzhou, LanZhou, Gansu Province, China
}

\begin{abstract}
To study what kind of role uric acid play on the relationship between oxidative Stress and inflammation in peripheral and cerebral system of oxonate-induced hyperuricemic rats. Twenty-six eight male Wistar rats were divided into two groups randomly. Potassium oxonate was used to establish hyperuricemic model for four weeks. In $2^{\text {nd }}$ and $4^{\text {th }}$ week, uric acid (UA) level, total superoxide dismutase (T-SOD), Gu, ZnSOD activity and interleukin-1beta (IL-1 $\beta$ ) concentration in serum were determined respectively. In $4^{\text {th }}$ week, one hour after last PO treatment, five rats of every group were given Evans Blue to test blood-brain barrier (BBB) permeability. Other brains were obtained to analysis T-SOD, Gu,Zn-SOD activity and IL-1 $\beta$ concentration in cerebral system. Meanwhile, brain and kidney were stained with hematoxylin and eosin $(\mathrm{H} \& \mathrm{E})$ to observe pathological change. In $2^{\text {nd }}$ week, both of T-SOD and Gu,Zn-SOD activity in serum increased obviously $(\mathrm{P}<0.05)$ in hyperuricemia rats. However, IL-1 $\beta$ content didn't change remarkably. In the $4^{\text {th }}$ week, T-SOD activity in model group had become similar with control group, and at the same time IL-1 $\beta$ content in serum increased significantly $(\mathrm{P}<0.05)$. Pathological section showed the structural and functional unit of the kidney had been damaged. On the contrary, both of T-SOD and Gu,Zn-SOD activity in brain increased obviously $(\mathrm{P}<0.05)$, but IL-1 $\beta$ concentration was no significant difference between two groups. In addition, the results of Evans Blue and H\&E suggested the integrity of BBB and structure of brain were not changed after PO treatment. The permeability of BBB and form of UA would be potential factors to decide what kind role UA play on keeping balance between antioxidative stress and induction of inflammatory response.
\end{abstract}

Keywords: Hyperuricemic. Blood brain barrier. Potassium oxonate. Oxidative stress. Inflammatory. Total-SOD. Gu,Zn-SOD. IL-1 $\beta$. Pathological injury.

\section{INTRODUCTION}

Uric acid (UA) is the end product of purine metabolism in humans. High UA level caused by metabolic disturbance would lead to hyperuricemia, which turns into risk factors to chronic renal disease (Feig, 2009), metabolic syndrome (Choi, Ford, 2007), hypertension (Mazzali et al., 2010) and cardiovascular disease (Grayson et al., 2011).

Owing to redundant UA deposition, tissue injury triggers innate responses and early phases of host defence, and promotes more pro-inflammatory cytokine producing,

\footnotetext{
*Correspondence: L. J. Ping. College of Veterinary Medicine, Gansu Agricultural University. No.1 Ying Men Cun, Lanzhou, 730000, China. E-mail: liangjp100@sina.com
}

initiating the inflammatory reaction. It was well known that pro-inflammatory cytokine has been closer link with nervous system disease, such as interleukin-1beta (IL-1 $\beta$ ), which could aggravate amyloid peptide deposits in brain and promote development of Alzheimer's disease (AD) (François et al., 2013).

It seems UA is a risk factor to central nervous system disease. However, as Alvarez-Lario said (Alvarez, Macarrón, 2011), if UA was a waste or harmful product, it poorly explained why our kidneys recover $90 \%$ of filtered UA, instead of eliminating it. In fact, UA as a natural antioxidant plays an important role not only on induction of inflammatory response but also on anti-oxidative stress in peripheral and central tissue (Glantzounis et al., 2005). Especially in cerebral system, UA have displayed protective effects on neurons, and might been correlated 
with a reduced risk of some neurodegenerative diseases including Parkinson's disease (PK) (Andreadou et al., 2009) and AD (Cutler et al.,2015).

Above-mentioned diseases have been reported to contribute to a high oxidative stress condition in internal organism environment. It is confused, in peripheral circulatory system, why high UA level complicated with those diseases can not exert antioxidant activity for organic tissues, but become inducing factor associated with inflammatory reaction. Up to now, it is not clear that how UA pretend neuron and cerebral tissue from superoxide and hydroxyl free radicals, instead of inducing inflammatory factors. Pakpoor reported the overall risk of subsequent multiple sclerosis (MS), PD and motor neuron disease (MND), which was significantly attributable to an increased risk observed in the early years after hospitalization for gout. Moreover after five years, the increased risk of neurological disease did not remain (Pakpoor et al., 2015).

Therefore, we postulated that it may exist some ways to assist UA to keep a balance between anti-oxidative stress and induction of inflammatory response. This researcher observed what kind of variety of oxidative stress and inflammation in peripheral and cerebral system under high UA condition, which would reveal the potential factors to destroy the balance.

\section{MATERIAL AND METHODS}

\section{Material}

Potassium oxonate and Evans Blue dye was purchased from Sigma Chemical Co. (St Louis, MO, USA); Uric acid and super oxide dismutase (SOD) assay kits were from Jiancheng Biotech, Nanjing, China; IL$1 \beta$ ELISA assay kit was obtained from Meilian Biotech, Shanghai, China. All other reagents used were from of analytical grades.

\section{Animal preparation}

Twenty-six eight male Wistar rats (8-10 weeks of age, weighing 200-220 g) were obtained from Experimental Animals Center in GanSu University of Chinese Medicine. They were fed with a commercial laboratory diet and allowed food and water ad libitum for an acclimatization period of 1 week prior to the experiment. Housing conditions and experimental procedures were set to be in accordance with international standards. All animals were maintained on a $12 \mathrm{~h}$ daynight cycle and the temperature and humidity were kept at $23 \pm 1{ }^{\circ} \mathrm{C}$ and $50 \%$, respectively. The animal model was made as described previously. Briefly, rats were divided into two groups randomly. One group as model group was injected with potassium oxonate (PO) $250 \mathrm{mg} / \mathrm{kg}$ intraperitoneally(i.p.), dissolved in normal saline (NS). And control group was administrated with equal volume NS. Treatments were carried out for four weeks after hyperuricemia induction (Haidari, Rashidi, Mohammad, 2012). Then all rats were sacrificed $1 \mathrm{~h}$ after last PO treatment.

\section{Assay for serum uric acid (SUA)}

Blood samples were obtained for SUA analysis in $2^{\text {nd }}$ and $4^{\text {th }}$ week. Whole blood samples were centrifuged for 5 $\mathrm{min}$ at $12000 \mathrm{rev} / \mathrm{min}$ to get serum samples. SUA levels were determined using enzymatic colorimetric methods, and measured by a spectrometer $(\lambda=690 \mathrm{~nm})$.

\section{Assay for Total superoxide dismutase (SOD) and $\mathrm{Gu}, \mathrm{Zn}-\mathrm{SOD}$ activity in brain and serum}

Blood samples were obtained for SOD analysis in $2^{\text {nd }}$ and $4^{\text {th }}$ week. When rats were sacrificed, left cerebral hemisphere were removed quickly, cleaned with cold phosphate buffer, and homogenized with NS (w/v, 1: 9) in a glass homogenizer. After centrifuging the homogenized brain tissue at $2500 \mathrm{rev} / \mathrm{min}$ for $15 \mathrm{~min}$, SOD activity of supernatant was measured by a spectrometer $(\lambda=550 \mathrm{~nm})$, and corrected by the protein content of per gram. Total $\mathrm{SOD}$ and $\mathrm{Gu}, \mathrm{Zn}-\mathrm{SOD}$ activity in serum were tested by the same way, which were expressed in $\mathrm{U} / \mathrm{ml}$ serum.

\section{ELISA to determine IL-1 $\beta$ concentration in brain and serum}

All samples prepared as described previously, ELISA assay experiments were conducted according to the manufacturer's instructions.

\section{Leakage of Evans Blue from brain vessels}

One hour after last PO treatment, Evans Blue $(80 \mathrm{mg} / \mathrm{kg})$ was injected into the rats' tail vein. Brain samples were taken one hour later. Five samples were needed. Brain cortex weighing $0.25 \mathrm{~g}$ was homogenized in 50\% trichloroacetic acid and centrifuged for $20 \mathrm{~min}$ at $1000 \mathrm{rev} / \mathrm{min}$. The resulting supernatants of the samples were diluted with ethanol, and the fluorescence of Evans Blue in brain cortex was detected by fluorescence spectrophotometry (excitation wavelength $620 \mathrm{~nm}$; 
emission wavelength $680 \mathrm{~nm}$ ). The concentrations of Evans Blue were calculated by standard curve. The results were expressed in microgram Evans Blue per gram cortex (Wu et al., 2009).

\section{Histological analysis}

When rats were sacrificed, right cerebral hemisphere and renal tissues were fixed in $10 \%(\mathrm{~V} / \mathrm{V})$ neutral buffered formalin for $24 \mathrm{~h}$, embedded in paraffin wax, cut into $4 \mu \mathrm{m}$ thicknesses, deparaffinized in xylene, and processed with graded ethanol series. Sections were stained with Hematoxylin and Eosin (H\&E) and observed by light microscopy (Leica DM500) at 200x magnification.

\section{Statistical analysis}

Data were represented as mean \pm standard deviation (SD), and analyzed by multifactorial analysis of variance, Student's t-test.

\section{RESULTS}

\section{Hyperuricemia model}

Treated with PO for 2 weeks, SUA level of rats was $70.15 \pm 7.64 \mu \mathrm{mol} / \mathrm{L}$ in control group and $83.22 \pm 17.56$ $\mu \mathrm{mol} / \mathrm{L}$ in model group $(\mathrm{n}=8)$. Compared with control rats injected with vehicle alone, PO made SUA level significantly increased by $18.6 \%(P<0.01)$ (Figure $1(\mathrm{a})$ ).

In $4^{\text {th }}$ week, SUA level of rats was $75.02 \pm 8.48$ $\mu \mathrm{mol} / \mathrm{L}$ in control group and $95.18 \pm 16.74 \mu \mathrm{mol} / \mathrm{L}$ in model group $(\mathrm{n}=8)$. As the same time, PO made SUA level significantly increased by $26.7 \%(P<0.01)$ (Figure $1(\mathrm{~b})$ ).

\section{Effect of hyperuricemia on T-SOD and Gu,Zn-SOD activity}

T-SOD and Gu, Zn-SOD activity in serum were measured in $2^{\text {nd }}$ and $4^{\text {th }}$ week, which evaluated antopxidation. In $2^{\text {nd }}$ week, T-SOD activity was $130.44 \pm 34.71 \mathrm{U} / \mathrm{mL}$ in control group and $187.21 \pm 21.31$ $\mathrm{U} / \mathrm{mL}$ in model group respectively. Gu,Zn-SOD activity was $80.40 \pm 34.71 \mathrm{U} / \mathrm{mL}$ in control group and $117.91 \pm 23.71$ $\mathrm{U} / \mathrm{mL}$ in model group respectively $(\mathrm{n}=8)$. Compared with control group, high UA level increased both T-SOD and Gu,Zn-SOD activity significantly $(P<0.01)$ (Figure 2(a)). In $4^{\text {th }}$ week, T-SOD activity was $121.34 \pm 12.17 \mathrm{U} / \mathrm{mL}$ in control group and $118.07 \pm 10.55 \mathrm{U} / \mathrm{mL}$ in model group respectively $(n=8)$. There was no significant difference between two groups. However, compared with control group $(79.95 \pm 14.21 \mathrm{U} / \mathrm{mL})$, the Gu,Zn-SOD activity in model group $(93.46 \pm 8.04 \mathrm{U} / \mathrm{mL})$ went up obviously $(P<0.05)$ (Figure 2(b)).

T-SOD and Gu,Zn-SOD activity in brain were measured in $4^{\text {th }}$ week. T-SOD level was $154.10 \pm 25.81 \mathrm{U} / \mathrm{g}$ in control group and $179.69 \pm 40.19 \mathrm{U} / \mathrm{g}$ in model group respectively $(\mathrm{n}=8)$. Gu,Zn-SOD activity was $44.55 \pm 12.72$ $\mathrm{U} / \mathrm{g}$ in control group and $111.24 \pm 31.47 \mathrm{U} / \mathrm{g}$ in model group respectively $(\mathrm{n}=8)$. Compared with control group, both T-SOD and $\mathrm{Gu}, \mathrm{Zn}$-SOD activity increased significantly $(P<0.05, P<0.01)$ (Figure 3).

\section{Effect of hyperuricemia on IL-1ß concentration}

IL- $1 \beta$ concentration in serum was measured in $2^{\text {nd }}$ and $4^{\text {th }}$ week, which estimated inflammatory response. In $2^{\text {nd }}$ week, IL-1 $\beta$ concentration was $60.79 \pm 15.71 \mathrm{ng} / \mathrm{mL}$ in control group and $64.22 \pm 18.01 \mathrm{ng} / \mathrm{mL}$ in model group respectively $(\mathrm{n}=8)$. There was no significant difference
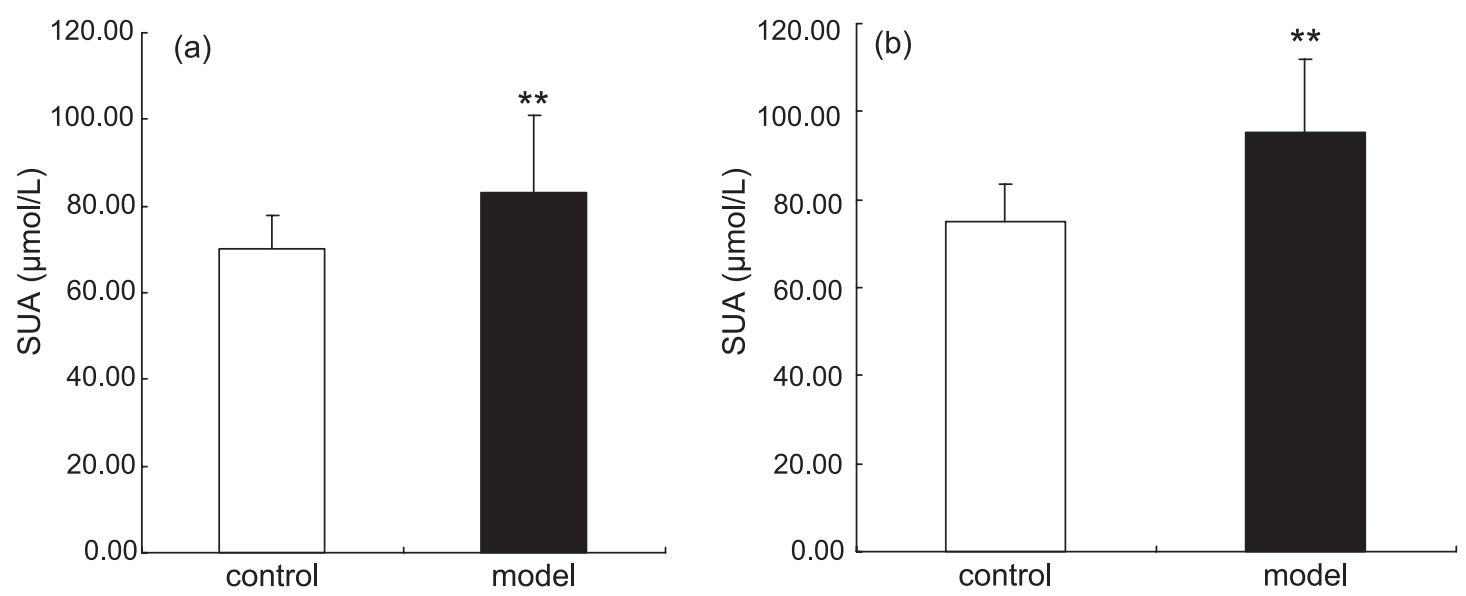

FIGURE 1 - Effect of PO treatment on rats' SUA in different time. Data are mean $\pm \mathrm{SD}, \mathrm{n}=12$. SUA, serum uric acid. (a): in $2^{\text {nd }}$ week and (b): in $4^{\text {th }}$ week. $* * P<0.01$ compared with control group 

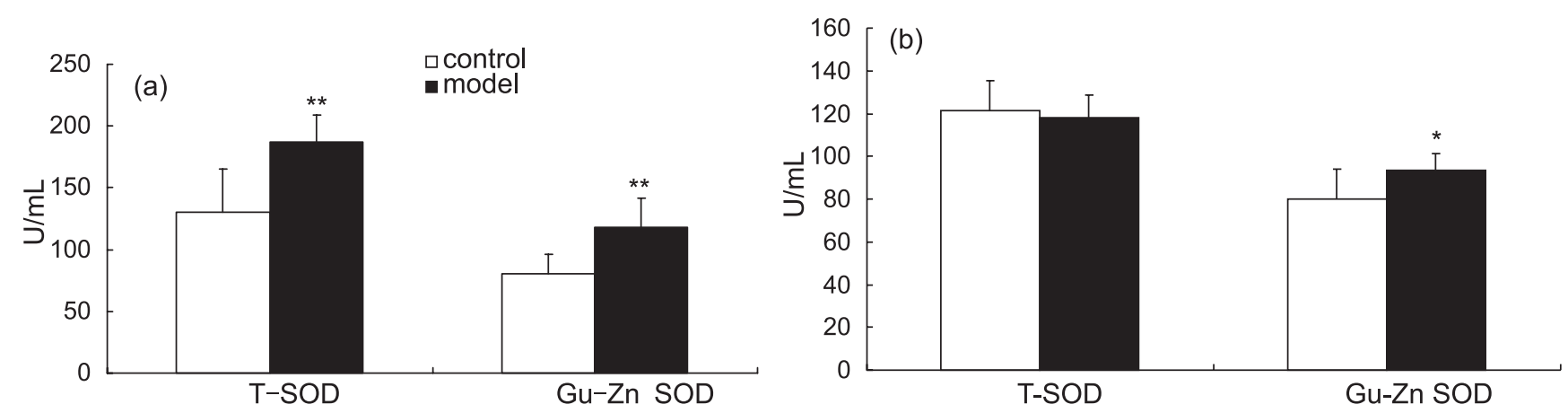

FIGURE 2 - Effects of hyperuricemia on T-SOD and Gu,Zn-SOD activity in serum in different time. Data are mean \pm SD, $n=8$. (a): in $2^{\text {nd }}$ week and (b): in $4^{\text {th }}$ week. $* P<0.05$, $* * P<0.01$ compared with control group

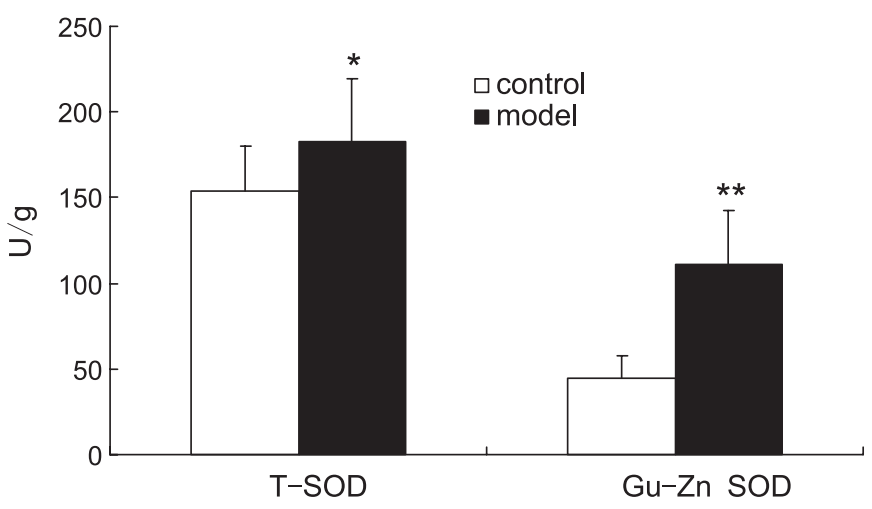

FIGURE 3 - Effects of hyperuricemia on T-SOD and Gu,Zn-SOD activity in brain in $4^{\text {th }}$ week. Data are mean $\pm \mathrm{SD}, \mathrm{n}=8 . * P<0.05$, $* * P<0.01$ compared with control group

between two groups $(P>0.05)$ (Figure $4(\mathrm{a}))$. In $4^{\text {th }}$ week, IL$1 \beta$ concentration was $59.34 \pm 19.40 \mathrm{ng} / \mathrm{mL}$ in control group and $79.18 \pm 17.20 \mathrm{ng} / \mathrm{mL}$ in model group respectively $(\mathrm{n}=8)$. Compared with control group, high UA level increased IL-1 $\beta$ concentration significantly $(P<0.05)$ (Figure $4(b)$ ).

IL- $1 \beta$ concentration in brain was measured in $4^{\text {th }}$ week. It was $976.49 \pm 272.26 \mathrm{pg} / \mathrm{g}$ in control group and $984.91 \pm 231.27 \mathrm{pg} / \mathrm{g}$ in model group respectively $(\mathrm{n}=8)$. There was no significant difference between two groups $(P>0.05)$ (Figure 5).

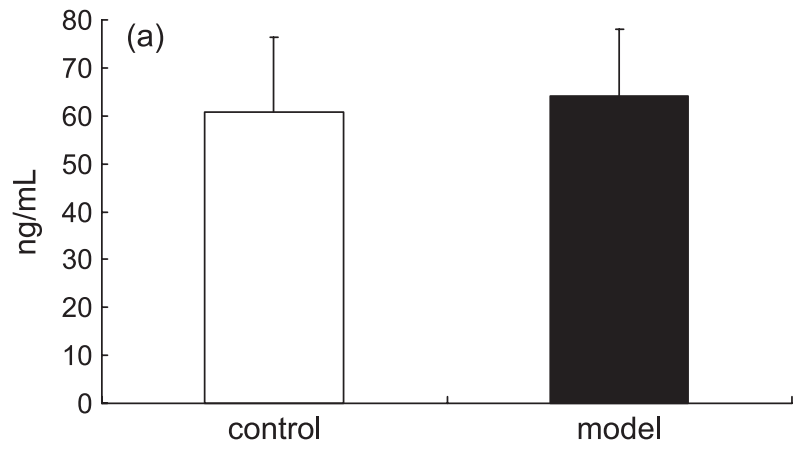

\section{Effect of hyperuricemia on permeability of the blood-brain barrier in rats}

Evans Blue dye was used as a blood-brain barrier indicator for examining the extravasation from the blood to the brain. The assay for leakage of Evans Blue from brain vessels showed that the dye concentration was $8.91 \pm 2.45$ $\mu \mathrm{g} / \mathrm{g}$ in control group and $8.54 \pm 2.02 \mu \mathrm{g} / \mathrm{g}$ in model group $(n=5)$. There was no significant difference between the two groups, which suggested the integrity of the blood-brain barrier was not changed after PO treatment.

\section{Effect of hyperuricemia on pathological section}

In control group, glomerular showed a sharply circumscribed boundary of tissue. Meanwhile the structure of renal tubular epithelium was clear and holonomic. No inflammatory exudate could be observed in renal interstitium. In model group, the structural and functional unit of the kidney was damaged. Glomerular contraction occurred, and the most striking change in the tubules was hydropic degeneration of the epithelium. Renal interstitium was collapsed and surrounded by inflammatory exudate with epithelial cells necrosis and abscission in nephron (Figure 6).

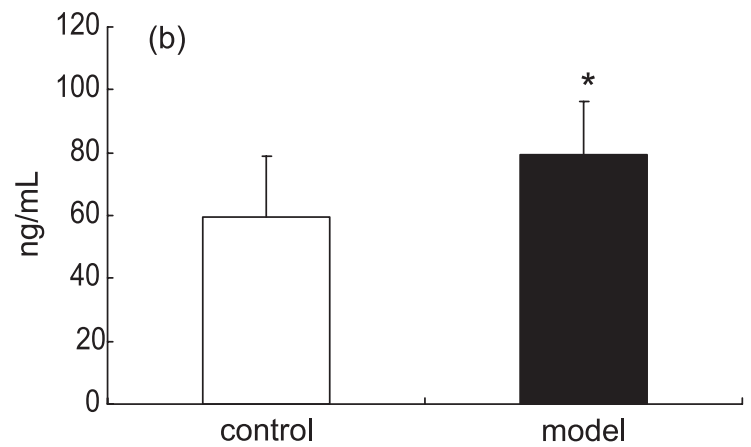

FIGURE 4 - Effects of hyperuricemia on IL-1 $\beta$ concentration in serum in different time. Data are mean $\pm S D, n=8$. (a): in $2^{\text {nd }}$ week and (b): in $4^{\text {th }}$ week. $* P<0.05$ compared with control group 


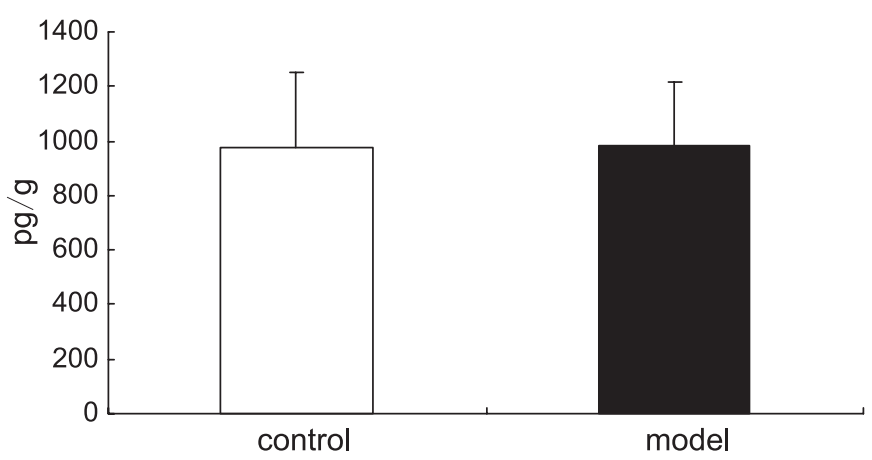

FIGURE 5 - Effects of hyperuricemia on IL-1 $\beta$ concentration in brain in $4^{\text {th }}$ week. Data are mean $\pm \mathrm{SD}, \mathrm{n}=8$.

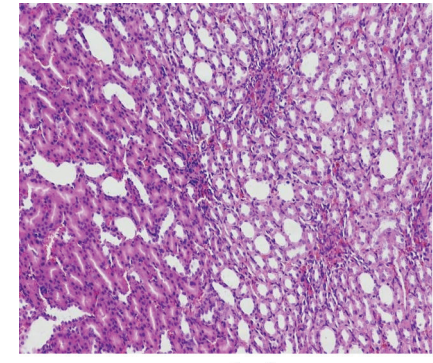

control

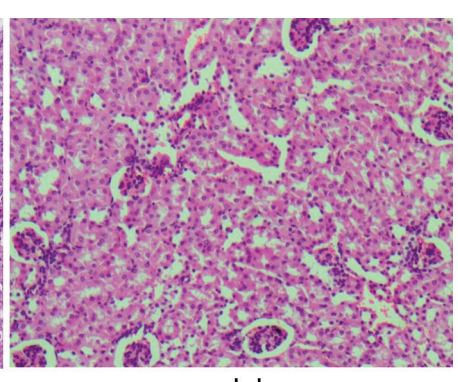

model
FIGURE 6 - Pathological section of renal tissue in $4^{\text {th }}$ week $(\mathrm{HE} \times 200)$.

However, no pathological injuries could be observed in brain of two groups (Figure 7).

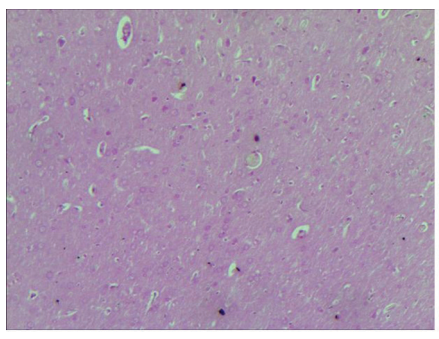

control

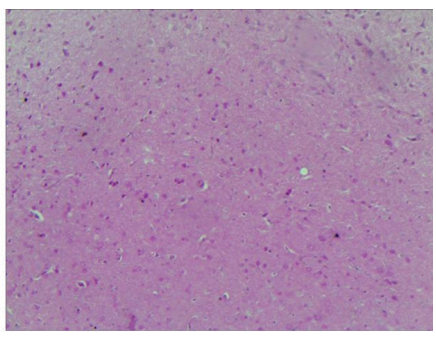

model
FIGURE 7 - Pathological section of brain in $4^{\text {th }}$ week $(\mathrm{HE} \times 100)$.

\section{DISCUSSION}

In peripheral system, our data show in the $2^{\text {nd }}$ week, compared with control group, both of T-SOD and $\mathrm{Gu}, \mathrm{Zn}$-SOD activity in serum increased obviously $(P<0.05)$ in hyperuricemia rats. However, IL-1 $\beta$ content didn't change remarkably. From $2^{\text {nd }}$ week, rats in model group drank more water and excreted more urine (Data not show), which meant the function of rats' kidney had been damaged. In the $4^{\text {th }}$ week, T-SOD activity in model group had become similar with control group, and meanwhile IL- $1 \beta$ content increased significantly
$(P<0.05)$. Pathological section showed basement membrane of kidney tubules and glomerulus had been destroyed under hyperuricemia condition. It predicted that organism produced anti-response to stimulation effect caused by initial high UA level and enhanced immune responses, while UA mainly played a role of antioxidant activity rather than induction of inflammatory response. Nevertheless, when high UA level sustained in peripheral system, UA would have displayed effects on induction of inflammatory response. Especially UA concentration in serum was high enough to form sodium urate crystals which produced a chronic inflammatory response with interstitial fibrosis and brought luxuriant pro-inflammatory cytokine such as IL-1 $\beta$ (Johnson et al., 1999).

It was worth to note that in the $4^{\text {th }}$ week, $\mathrm{Gu}, \mathrm{Zn}-\mathrm{SOD}$ activity in model group was still higher than control. However, UA didn't take on adequate antioxidation against inflammatory reaction induced by IL-1 $\beta$.

In Cerebral system, T-SOD activity increased obviously $(\mathrm{P}<0.05)$ in the brain of rats with hyperuricemia. Yet there was no difference between two groups in IL-1 $\beta$ content. The assay for leakage of Evans Blue dye from brain vessels and pathological section of brain showed that the blood-brain barrier (BBB) permeability was not damaged in the model group, which signified that UA had not mad microvascular endothelial cell in brain injured. It conjectured that BBB kept IL- $1 \beta$ originated from peripheral system out, and defended cerebral tissue from inflammatory response. Therefore, UA as an antioxidant protected brain tissue and neurons in the early stage.

It is known there are three types of mitochondrial antioxidant enzyme, including copper, zinc superoxide dismutase ( $\mathrm{Cu}, \mathrm{Zn}-\mathrm{MOD})$, manganese superoxide dismutase (Mn-SOD) and iron superoxide dismutase (Fe-SOD). All of them are crucial in maintaining cellular and organism homeostasis, which are tightly regulated during inflammatory challenges. Qiu X found that induction of Mn-SOD gene expression by IL- $1 \beta$ had been mediated through a complex intronic enhancer element (Tong et al., 2005). T-SOD and Cu,Zn-MOD activity can reflect action of proinflammatory cytokine indirectly. From our researches, both in peripheral and cerebral system SOD activity was influenced mainly by UA instead of IL-1 $\beta$, which exhibited protection.

According to our data, mechanism of central protection may involve two ways. One was permeability of $\mathrm{BBB}$, and another was the form of UA. It is well known the $\mathrm{BBB}$ is important for the maintenance of water and protein balance between the intravascular and extravascular compartments. Any injuries in 
endothelial barrier function implicated in the genesis and/or progression of a variety of pathological conditions would lead to neurodegenerative disorders, angioedema, sepsis and cancer (Rodrigues, Granger, 2015). Proinflammatory cytokine from peripheral system might affect the function of the central nervous system (CNS) by crossing the BBB for direct interaction with CNS tissue. Saturable transport systems from blood to the CNS have been described for interleukin-1 alpha (IL$1 \alpha$ ), IL-1 $\beta$, IL-1 receptor antagonist (IL-1 ra), and tumor necrosis factor-alpha (TNF- $\alpha$ ). There is a large number of blood-to-brain uptakes of IL-1, IL-1 $\beta$, and IL-1 ra located in the most brain sites, which make incidence rate of inflammatory reaction reduced (Banks, Kastin, Broadwell, 1995). It is reported a pro-inflammatory cytokine response is associated with greater clinical severity, BBB permeability, and neuroimaging damage in encephalitis (Michael et al., 2015). Sadowska found Blood-to-brain transport of IL- $1 \beta$ was higher across brain regions in fetuses exposed to ischemia-reperfusion than non-ischemic fetuses. Therefore, hypoxic-ischemic injury could accentuate systemic cytokine transfer across the fetal BBB (Sadowska et al., 2015). Tong $\mathrm{XK}$ reported oxidative stress and structural alterations in the cerebrovascular dysfunctions were associated with AD (Qiu et al., 2008). If permeability of BBB had been damaged, it would become more convenient for redundant pro-inflammatory cytokine cross $\mathrm{BBB}$ to enter cerebrospinal fluid and interstitial fluid spaces of the brain and spinal cord.

So we considered that UA could play a role on neuron protection in brain instead of induction of inflammatory response, when overfull UA hadn't made permeability of BBB damaged. How does UA work? The form of UA may be the crucial factor.

In summary, BBB is a significant barrier to limit cytokine (e.g.IL-1 $\beta$ ) invading into brain. Furthermore, $\mathrm{BBB}$ also restricts UA level in brain. In other words, whether UA can cross BBB and show antioxidation has been decided by the form itself. Firstly, only water-soluble and low molecular weight (LMN) compounds, such as morphine, are known to cross the BBB in sufficient amounts (Banks, Kastin, Broadwell, 1995). Free UA with LMN is slightly soluble in water. Only a small quantity of UA should be allowed to cross BBB and enter brain from peripheral system, which improves SOD activity in order to clean more reactive oxygen species (ROS). In the meanwhile, it still significantly decreased apoptosis via ROS formation and inflammatory cytokine production in CNS (Barichello et al., 2011). Even though UA formed crystal, holonomic BBB would be tight enough to keep them away from brain and prevent crystal deposition on tissue surfaces. In phagocytes, UA crystals have also been shown to trigger stress signals, including generation of free radicals, potassium efflux and cathepsin B release from fractured liposomes, which may be a key in the activation of the inflammasome (Martinon, 2010).

Secondly, both UA and metabolite of UA own antioxidation. Haberman reported the synthetic 1,7-dimethyl derivative of UA reported previously to be protective in models of brain focal ischemia in mice retained the protective effects of its parent compound (Haberman et al., 2007). But not all catabolites evoke antioxidative effect. In Guerreiro's researches, xanthine the immediate precursor of UA, which was produced via the degradation pathway of adenosine or that of guanosine, failed to afford neuroprotection. Likewise, hypoxanthine and guanine, the two purines bases, which gave rise to xanthine via catabolism of adenosine and guanosine, respectively, were ineffective and failed to reduce intracellular oxidative stress too (Guerreiro et al., 2009). And they found there was no positive correlation between UA concentration and antioxidation. Neuroprotection by UA was reproduced by desferrioxamine $(10 \mu \mathrm{M})$ which predominantly chelates ferric iron and by catalase $(500$ $\mathrm{IU} / \mathrm{mL})$. Nevertheless, the survival effect of UA $(200 \mu \mathrm{M})$ was not improved by any of these antioxidants (Guerreiro et al., 2009).

\section{CONCLUSION}

In the pathological process of hyperuricemia, UA level mainly played a role on antioxidant activity rather than induction of inflammatory response in the beginning. As high UA level sustained in peripheral system until organ tissue damaged by sodium urate crystals, UA would display effects of induction of inflammatory response. However, in Cerebral system, if BBB kept integrity, UA would protect brain tissue and neurons as an antioxidant. In view of the above, no matter in peripheral or cerebral system, UA could play an important role both on antioxidative stress and induction of inflammatory response. The permeability of BBB and form of UA would be potential factors to decide which action of UA occupied predominant position and keep balance between protection and damage.

\section{ACKNOWLEDGEMENT}

This work was supported by the grant of LanZhou City Talent Innovation and Entrepreneurship Project (No. 2015-RC-22). 


\section{CONFLICT OF INTEREST}

The Author(s) declare(s) that they have no conflicts of interest to disclose.

\section{REFERENCE}

Alvarez-Lario B, Macarrón-Vicente J. Is there anything good in uric acid? QJM. 2011;104(12):1015-1024.

Andreadou E, Nikolaou C, Gournaras, Rentzos M, Boufidou F, Tsoutsou AF, et al. Serum uric acid levels in patients with Parkinson's disease: their relationship to treatment and disease duration. Clin Neurol Neurosurg. 2009;111(9):724-728.

Banks WA, Kastin AJ, Broadwell RD. Passage of cytokines across the blood-brain barrier. Neuroimmunomodulation. $1995 ; 2(4): 241-8$.

Barichello T, Lemos JC, Generoso JS, Cipriano AL, Milioli GL, Marcelino DM, et al. Oxidative stress, cytokine/chemokine and disruption of blood-brain barrier in neonate rats after meningitis by Streptococcus agalactiae. Neurochem Res. 2011;36(10):1922-1930.

Choi HK, Ford ES. Prevalence of the metabolic syndrome in individuals with hyperuricemia. Am J Med. 2007;120(5):442447.

Cutler RG, Camandola S, Malott KF, Edelhauser MA, Mattson MP. The role of uric acid and methyl derivatives in the prevention of age-related neurodegenerative disorders. Curr Top Med Chem. 2015;15(21):2233-8.

Feig DI. Uric acid: a novel mediator and marker of risk in chronic kidney disease? Curr Opin Nephrol Hypertens. 2009;18(6):526-530.

François A, Terro F, Janet T, Rioux Bilan A, Paccalin M, Page G. Involvement of interleukin- $1 \beta$ in the autophagic process of microglia: relevance to Alzheimer's disease. J Neuroinflammation. 2013;10:151.

Glantzounis G, Tsimoyiannis EC, Kappas AM, Galaris DA. Uric acid and oxidative stress. Curr Pharm Des. 2005;11(32):41454151.

Grayson PC, Kim SY, Lavalley M, Choi HK. Hyperuricemia and incident hypertension: a systematic review and meta-analysis. Arthritis Care Res. 2011;63(1):102-110.
Guerreiro S, Ponceau A, Toulorge D, Martin E, Alvarez-Fischer D, Hirsch EC, et al. Protection of midbrain dopaminergic neurons by the end-product of purine metabolism uric acid: potentiation by low-level depolarization. J Neurochem. 2009;109(4):1118-1128.

Haberman F, Tang SC, Arumugam TV, Hyun DH, Yu QS, Cutler RG, et al. Soluble neuroprotective antioxidant uric acid analogs ameliorate ischemic brain injury in mice. Neuromol Med. 2007;9(4):315-323.

Haidari F, Rashidi MR, Mohammad-Shahi M. Effects of orange juice and hesperetin on serum paraoxonase activity and lipid profile in hyperuricemic rats. BioImpacts. 2012;2(1):39-45.

Johnson RJ, Kivlighn SD, Kim YG, Suga S, Fogo AB. Reappraisal of the pathogenesis and consequences of hyperuricemia in hypertension, cardiovascular disease, and renal disease. Am J Kidney Dis. 1999;33(2):225-34.

Martinon F. Update on biology: uric acid and the activation of immune and inflammatory cells. Curr Rheumatol Rep. 2010;12(2):135-141.

Mazzali M, Kanbay M, Segal MS, Shafiu M, Jalal D, Feig DI, Johnson RJ. Uric acid and hypertension: cause or effect? Curr Rheumatol Rep. 2010;12(2):108-117.

Michael BD, Griffiths MJ, Granerod J, Brown D, Keir G, Wnęk $\mathrm{G}$, et al. The interleukin-1 balance is associated with clinical severity, blood-brain barrier permeability, neuroimaging changes and outcome in encephalitis. J Infect Dis. 2015;213(10):16511660.

Pakpoor J, Seminog OO, Ramagopalan SV, Goldacre MJ. Clinical associations between gout and multiple sclerosis, Parkinson's disease and motor neuron disease: record-linkage studies. BMC Neurol. 2015;15(16):1-6.

Qiu X, Aiken KJ, Chokas AL, Beachy DE, Nick HS. Distinct functions of CCAAT enhancer-binding protein isoforms in the regulation of manganese superoxide dismutase during interleukin-1beta stimulation. J Biol Chem. 2008;283(38):2577425785 .

Rodrigues SF, Granger DN. Blood cells and endothelial barrier function. Tissue Barriers. 2015;3(3):1-2. 
Sadowska GB, Chen X, Zhang J, Lim YP, Cummings EE, Makeyev $\mathrm{O}$, et al. Interleukin- $1 \beta$ transfer across the bloodbrain barrier in the ovine fetus. J Cereb Blood Flow Metab. 2015;35(9):1388-1395.

Tong XK, Nicolakakis N, Kocharyan A, Hamel E. Vascular remodeling versus amyloid beta-induced oxidative stress in the cerebrovascular dysfunctions associated with Alzheimer's disease. J Neurosci. 2005; 25(48):11165-11174.
Wu J, Ji H, Wang YY, Wang, Li YQ, Li WG, Y et al. Glutathione depletion upregulates P-glycoprotein expression at the bloodbrain barrier in rats. J Pharm Pharmacol. 2009;61(6):1-6.

Received for publication on $04^{\text {th }}$ December 2016

Accepted for publication on $12^{\text {th }}$ April 2017 